\title{
Relación del doble estándar sexual con el funcionamiento sexual y las conductas sexuales de riesgo: revisión sistemática
}

\author{
Ana Álvarez-Muelas, Carmen Gómez-Berrocal y Juan Carlos Sierra* \\ Centro de Investigación Mente, Cerebro y Comportamiento (CIMCYC), Universidad de Granada, España \\ - Recibido: 04 - 04 - 2020 . Aceptado: 17 - 04 - 2020 . Avance online: 28 - 05 - 2020
}

RESUMEN. El doble estándar sexual (DES) hace referencia a la aceptación de diferentes criterios para la valoración del mismo comportamiento sexual en hombres y en mujeres. Ante la ausencia de revisiones bibliográficas previas, este trabajo presenta una revisión sistemática sobre la relación del DES con el funcionamiento sexual y las conductas sexuales de rie sgo. A partir de la búsqueda en diferentes bases de datos, se obtuvieron 22 artículos científicos. Los resultados señalan que la actitud a favor del DES perjudica el funcionamiento sexual y favorece las conductas sexuales de riesgo, las cuales son más perjudiciales para las mujeres. Se discute la importancia que tiene el estudio del DES en la salud sexual, para entender el funcionamiento sexual y la incidencia de las conductas sexuales de riesgo. Se recomienda mejorar los instrumentos para medir DES, con el fin de garantizar una evaluación exacta e invariante de este fenómeno en aquellos grupos definidos por el género y otras variables socioculturales.

PALABRAS CLAVE: Doble estándar sexual, Funcionamiento sexual, Conductas sexuales de riesgo, Salud sexual, Revisión sistemática.

Relationship of sexual double standard with sexual functioning and risk sexual behaviors: A systematic review ABSTRACT. Sexual double standard (SDS) refers to the acceptance of different criteria for the assessment of the same sexual behavior in men and women. Due to the absence of previous bibliographical reviews, this study presents a systematic review of the relationship between SDS, sexual functioning, and risk sexual behaviors. From a search of different databases, it was obtained a total of 22 scientific articles. The results indicate that attitude in favor of SDS harms sexual functioning and favors risk sexual behaviors, which were more harmful to women. The importance of the study of SDS in sexual health is discussed, to understand sexual functioning and the incidence of risk sexual behaviors. It is recommended to improve the instruments used to measure SDS, to guarantee an exact and invariant evaluation of this phenomenon in those groups defined by gender and other socio-cultural variables.

KEYWORDS: Sexual double standard, Sexual function, Risk sexual behaviors, Sexual health, Systematic review.

El doble estándar sexual (DES) hace referencia a la aceptación de diferentes criterios para la valoración del mismo comportamiento sexual en hombres y en mujeres (Milhausen y Herold, 2001). El DES tradicional acepta mayor libertad sexual para el hombre que para la mujer,

\footnotetext{
*Correspondencia: Juan Carlos Sierra.

Facultad de Psicología, Campus Universitario de Cartuja, s/n. C.P: 18011 , Granada, España.

E-mail: jcsierra@ugr.es
}

(C) 2020 Sociedad Universitaria de Investigación en Psicologíay Salud. Publicado por Consejo General de Colegios Oficiales de Psicólogos, España. Este es un artículo Open Access
bajo la CC BY-NC-ND licencia (http://creativecommons.org/licencias/by-nc-nd/4.0/). lo que conlleva que ciertas conductas sexuales sean mejor valoradas en ellos que en ellas. Esta actitud se ha encontrado tanto en hombres como en mujeres, aunque con mayor prevalencia en los hombres (Álvarez-Muelas, Gómez-Berrocal, Vallejo-Medina, y Sierra, 2019; Guo, 2019; Gutiérrez-Quintanilla, Rojas-García, y Sierra, 2010; Sánchez-Fuentes, Moyano, GómezBerrocal, y Sierra, 2020; Sierra, Moyano, Vallejo-Medina, y Gómez- Berrocal, 2018). Se ha descrito prácticamente en todas las edades, concretamente en adolescentes (Monge, Sierra, 
y Salinas, 2013), jóvenes adultos (GutiérrezQuintanilla et al., 2010; Sakaluk y Milhausen, 2012) y personas mayores (Sánchez-Fuentes et al., 2020; Sierra, Monge, Santos-lglesias, Rodríguez, y Aparicio, 2010), observándose una mayor aceptación del DES a medida que aumenta la edad (Sierra et al., 2018; Sprecher, 1989).

El estudio del DES se ha enmarcado en el contexto de la salud sexual, que es definida como la capacidad de hombres y mujeres para expresar libremente su sexualidad sin riesgo de enfermedades de transmisión sexual, embarazos no deseados, coerción, violencia y discrminación (Lottes, 2000). El funcionamiento sexual (FS) y las conductas sexuales de riesgo (CSR) constituyen dimensiones fundamentales de la salud sexual. El FS, entendido como respuesta sexual, incluye las dimensiones de deseo, exitación, orgasmo y satisfacción sexual (Carrobles y Sanz, 1991). Estas dimensiones están determinadas por factores biológicos, psicológicos, sociales y culturales (Arcos-Romero y Sierra, 2018; Calvillo, Sánchez-Fuentes, y Sierra, 2018; Sánchez-Fuentes, Santos-Iglesias, y Sierra, 2014). Por lo tanto, cabe pensar que las dimensiones del FS puedan verse afectadas por una actitud favorable al DES tradicional. Las CSR constituyen manifestaciones de la actividad sexual que aumentan la probabilidad de sufrir consecuencias negativas (PawlowskyGlahn, Egozcue, y Planes-Pedra, 2018), como pueden ser el contagio por el Virus de la Inmunodeficiencia Humana, otras infecciones de transmisión sexual (ITS) y embarazos no deseados. Las CSR más destacables son la participación en encuentros sexuales sin métodos de barrera-anticoncepción (Turchik y Gidycz, 2012), bajo el efecto del alcohol/ drogas (Isorna Folgar, Fariña Rivera, Sierra, y Vallejo-Medina, 2015) o con una persona diagnosticada de ITS (Senn, Carey, Vanable, Coury-Doniger, y Urban, 2007). Se sabe que un factor clave en el desarrollo de prácticas sexuales sin protección son las desigualdades de género (Harrison, $O$ 'Sullivan, Hoffman, Dolezal, y Morrell, 2006), y por ello las CSR pueden estar relacionadas con DES.

En el marco de lo que se ha expuesto sobre la posible relación de las actitudes a favor del DES tradicional con el FS y las CSR como dimensiones de la salud sexual, y ante la ausencia de revisiones sistemáticas previas, el objetivo del presente trabajo es llevar a cabo una revisión sistemática de los estudios que examinan la relación del DES con el FS y las CSR, por ende, de las implicaciones de estos constructos en la salud sexual de las personas.

\section{MÉTODO}

\section{•BÚSQUEDA DE ESTUDIOS}

La revisión sistemática siguió los pasos establecidos en la declaración PRISMA (Liberati et al., 2010). La búsqueda bibliográfica se realizó a través de las bases de datos Scopus, Web of Science, Psyclnfo y PsycArticles durante los meses de diciembre de 2019 a enero de 2020. Se limitó por título, resumen, palabras clave y/o tema. Los términos empleados fueron "sex* double* standard*" AND (sex* desire* OR sex* arousal* OR orgasm* OR sex* satisfact* OR sex* response* OR sex* funct*) OR ("aid*" OR "hiv" OR "std*" OR "sti*" OR "vener* diseas*" OR "unprotect* sex*" OR "unsaf* sex*" OR "sex* transmi*" OR "sex* infect*" OR "sex* ill*" OR "sex* deseas*" OR sex* risk* OR risk* OR risk* behav*), utilizando el truncamiento * para obtener cualquier variante de los términos con sus diferentes sufijos, y restringiéndose el tipo de documento a artículos científicos y a los idiomas español, inglés y portugués.

\section{• CRITERIOS DE INCLUSIÓN}

Las publicaciones seleccionadas cumplían con los siguientes criterios de inclusión:

1. La definición de DES implica diferencias en la valoración de comportamientos sexuales entre hombres y mujeres, resultando más favorable para el hombre (DES tradicional).

2. Estudian la relación del DES con el FS (deseo sexual, excitación sexual, orgasmo y/o satisfacción sexual), y/o con las CSR, tales como mantener relaciones sexuales sin métodos de barrera-anticoncepción, con un compañero/a sexual con ITS y/o padecer ITS. 
3. Evaluación del DES mediante instrumentos estandarizadas, ítems derivados de escalas estandarizadas o cuestionarios, entrevistas o ítems ad hoc.

\section{-SELECCIÓN Y CODIFICACIÓN DE LOS ESTUDIOS}

En primer lugar, se llevó a cabo una lectura del título y del resumen de todos los trabajos seleccionados. A continuación, se realizó la lectura comprensiva y su selección en función de los criterios de inclusión. Los artículos recopilados se examinaron a texto completo extrayendo la información:

- Título, autor/es y año de publicación.

- Número de participantes, porcentaje de hombres y de mujeres, edad y media de edad.

- Instrumentos de evaluación del DES.

- Resultados de la relación del DES tradicional con FS y CSR.

Se evaluó la calidad metodológica de los estudios seleccionados a través de la Quality Assesment Tool for Observational Cohort and Cros-Sectional Studies (National Heart, Lung, and Blood Institute, 2014) para los estudios con metodología cuantitativa, y el Strenghtening the Reporting of Observational studies in Epidemiology (van Elm et al., 2008) para los trabajos de metodología analítica observacional.

\section{- FIABILIDAD DE LA CODIFICACIÓN}

La codificación puede ser evaluada en términos de acuerdos inter-codificadores e intracodificadores con la kappa verdadera $\left({ }^{-} \kappa\right)$, corrigiendo el acuerdo con la verificación de la exacta correspondencia en la codificación (Fariña, Arce, y Novo, 2002; Morales-Toro, Guillén-Riquelme, y Quevedo-Blasco, 2019). En nuestro caso, los estudios se codificaron por las variables analizadas en la revisión, examinando semanas después los acuerdos intra-codificadores. El codificador volvió a codificar el $50 \%$ de los artículos $(n=11)$. La codificación fue evaluada mediante el análisis de categorías, codificando como acuerdo si en los momentos 1 y 2 registraba las mismas categorías, y como desacuerdo si registraba distintas categorías. Los resultados mostraron una concordancia intra-codificador totalmente coincidente para las variables título, autor/es, año de publicación, instrumentos de evaluación y resultados, y muy buena para la categoría de participantes $\left({ }^{-} \kappa=0,88\right)$. Por tanto, la codificación es fiable, es decir, otro codificador entrenado hubiera elaborado una base de datos similar (Monteiro, Vázquez, Seijo, y Arce, 2018).

\section{RESULTADOS}

Se obtuvo un total de 171 trabajos, de los cuales se eliminaron 56 duplicados. De los 115 estudios resultantes, se eliminaron todos los que no cumplieron los criterios de inclusión. Finalmente, los 22 artículos restantes fueron revisados bajo los estándares de calidad metológica, y todos se sometieron a la revisión sistemática. De estos trabajos, seis se centraban en la relación entre DES y FS, y diecisiete en la relación del DES con CSR (véase la Figura 1).

\section{- PARTICIPANTES}

En catorce de los trabajos incluyeron en sus muestras a hombres y a mujeres, siete exclusivamente a mujeres y un solo estudio incluyó únicamente a hombres. En cuanto a la edad, en cuatro estudios la muestra estaba formaba exclusivamente por adolescentes (12-18 años de edad), en seis estudios por adolescentes y adultos jóvenes (14-30 años de edad), en seis por adultos jóvenes (18-35 años de edad) y en cinco se incluyen adultos (18-74 años de edad). Únicamente un trabajo incluyó a adolescentes y adultos (16-49 años de edad).

\section{- INSTRUMENTOS PARA EVALUAR EL DOBLE ESTÁNDAR SEXUAL}

En ocho trabajos se emplearon entrevistas abiertas y semiestructuradas, y en siete estudios se evaluó el DES a través de cuestionarios y preguntas ad hoc. En los siete trabajos restantes se utilizaron instrumentos estandarizados: en cinco la Double Standard Scale (DSS; Caron, Davis, Halteman, y Stickle, 1993, 1998), en uno el Sexual Double Standard Evaluation 


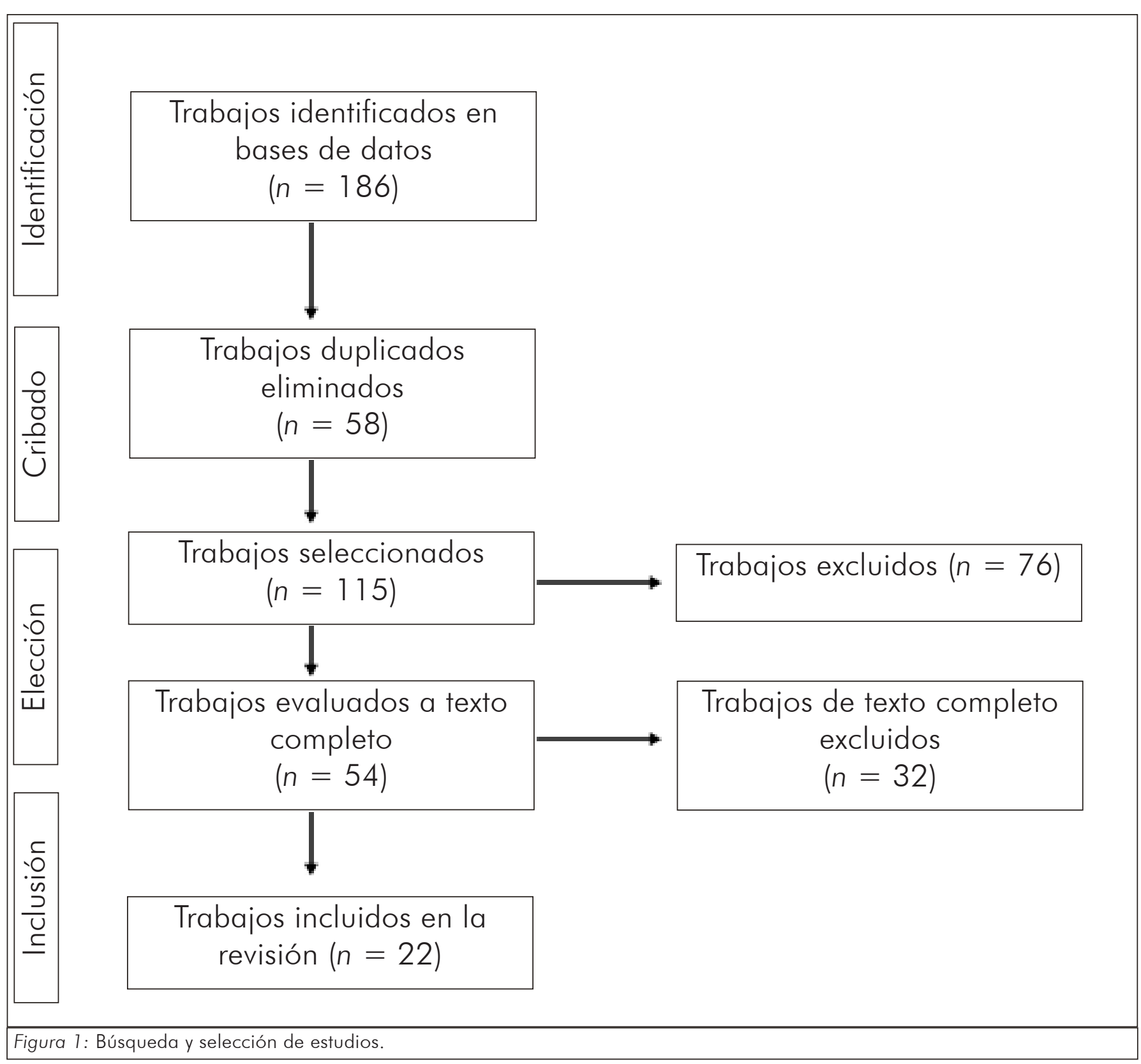

(Milhausen y Herold, 2001) y en otro la Sexual Double Standard Scale (SDSS; Muehlenhard y Quackenbush, 2011).

\section{- RELACIÓN DEL DOBLE ESTÁNDAR SEXUAL CON EL FUNCIONAMIENTO SEXUAL}

Sobre los trabajos de la relación DES-FS, dos se centraron en el deseo sexual, uno en el orgasmo y cuatro en la satisfacción sexual. El apoyo al DES dificultó en las mujeres la experiencia y el reconocimiento del deseo sexual, y el alcance de la experiencia orgásmica (Jackson y Cram, 2003; Kelly et al., 2017). Con respecto a la relación entre DES y satisfacción sexual, se encontró que la satisfacción sexual se asocia de forma negativa con el DES en hombres y mujeres
(Haavio-Mannila y Kontula, 2003; Horne y Zimmer-Gembeck, 2006; Santos-Iglesias et al., 2009). En el estudio de Marques, Viera y Pechorro (2013), cuya muestra estaba integrada exclusivamente por hombres, no se halló relación entre ambas variables (véase Tabla 1).

\section{- RELACIÓN DEL DOBLE ESTÁNDAR SEXUAL CON CONDUCTAS SEXUALES DE RIESGO}

Los resultados indican que la actitud de las mujeres a favor del DES se relaciona de forma directa con el menor uso de protección en las relaciones sexuales (Danube et al., 2015; Lotfi, Tehrani, Khoei, Yaghmaei, y Dworkin, 2013; Teitelman, Tennille, Bohinski, Jemmott, y Jemmott, 2013). Solo el estudio de Srijaiwong, Sinshu, 
Tabla 1

Estudios sobre la relación del doble estándar sexual (DES) y el funcionamiento sexual

\begin{tabular}{|c|c|c|c|c|}
\hline Autor/es & $\begin{array}{l}\text { Variables } \\
\text { relacionadas } \\
\text { con el DES }\end{array}$ & Participantes & $\begin{array}{l}\text { Instrumentos } \\
\text { para evaluar } \\
\text { DES }\end{array}$ & Resultados \\
\hline $\begin{array}{l}\text { Haavio- Mannila } \\
\text { y Kontula (2003) }\end{array}$ & $\begin{array}{l}\text { Satisfacción } \\
\text { sexual }\end{array}$ & $\begin{array}{l}8.946 \text { participantes: } \\
41,50 \% \text { hombres y } \\
58,50 \% \text { mujeres } \\
\text { menores de } 74 \text { años } \\
\text { de edad* }\end{array}$ & Pregunta ad hoc. & $\begin{array}{l}\text { Los niveles de satisfacción sexual } \\
\text { se ven afectados por las normas } \\
\text { sexuales (DES) }\end{array}$ \\
\hline $\begin{array}{l}\text { Horne y Zimmer- } \\
\text { Gembeck (2006) }\end{array}$ & $\begin{array}{l}\text { Satisfacción } \\
\text { sexual }\end{array}$ & $\begin{array}{l}449 \text { mujeres de } 16 \text { a } \\
22 \text { años }(M=17,9)\end{array}$ & $\begin{array}{l}\text { Double Standard } \\
\text { Scale (DSS; Caron } \\
\quad \text { et al., 1993) }\end{array}$ & $\begin{array}{c}\text { La percepción de la habilidad para } \\
\text { alcanzar la satisfacción sexual se } \\
\text { correlaciona positivamente con la } \\
\text { actitud en } \\
\text { contra del DES }\end{array}$ \\
\hline $\begin{array}{l}\text { Jackson y Cram } \\
\qquad(2003)\end{array}$ & Deseo sexual & $\begin{array}{l}101 \text { hombres y } \\
\text { mujeres de } 16 \text { a } 18 \\
\text { años* }\end{array}$ & Entrevista & $\begin{array}{l}\text { EI DES favorece que las mujeres } \\
\text { tengan un escaso conocimiento } \\
\text { del deseo sexual. El deseo sexual } \\
\text { no se describe como experiencia } \\
\text { corporal ni se mencionan } \\
\text { alternativas al coito, como la } \\
\text { masturbación o el sexo oral }\end{array}$ \\
\hline Kelly et al. (2017) & $\begin{array}{l}\text { Deseo sexual. } \\
\text { Orgasmo. }\end{array}$ & $\begin{array}{l}94 \text { mujeres de } 16 \text { a } \\
49 \text { años de edad* }\end{array}$ & Entrevista & $\begin{array}{c}\text { El DES impregna las prácticas } \\
\text { sexuales y anticonceptivas de } \\
\text { las mujeres, centrándolas en el } \\
\text { placer y el deseo de los hombres, } \\
\text { y otorgando poca importancia a la } \\
\text { experiencia orgásmica }\end{array}$ \\
\hline $\begin{array}{l}\text { Marques, Viera y } \\
\text { Pechorro (2013) }\end{array}$ & $\begin{array}{l}\text { Satisfacción } \\
\text { sexual }\end{array}$ & $\begin{array}{c}262 \text { hombres } \\
\text { divididos en dos } \\
\text { grupos de edad: } 132 \\
\text { participantes de } 20- \\
30 \text { años de edad ( } M \\
=23,88 \text { ) y } 130 \text { de } \\
40-50 \text { años ( } M= \\
46,27)\end{array}$ & $\begin{array}{l}\text { Sexual Double } \\
\text { Standard } \\
\text { Evaluation } \\
\text { (Milhausen y } \\
\text { Herold, 2001; } \\
\text { Leal, Carvalho y } \\
\text { Ramos, 2005) }\end{array}$ & $\begin{array}{c}\text { La satisfacción sexual no } \\
\text { correlaciona con la aceptación del } \\
\text { DES }\end{array}$ \\
\hline $\begin{array}{l}\text { Santos- Iglesias et } \\
\text { al. (2009) }\end{array}$ & $\begin{array}{l}\text { Satisfacción } \\
\text { sexual }\end{array}$ & $\begin{array}{l}646 \text { participantes: } \\
45,8 \% \text { hombres y } \\
54,2 \% \text { mujeres de } \\
18 \text { a } 58 \text { años de } \\
\text { edad }(M=26,40)\end{array}$ & $\begin{array}{l}\text { Double Standard } \\
\text { Scale (DSS; Caron } \\
\quad \text { et al., 1993) }\end{array}$ & $\begin{array}{l}\text { El DES correlacionó en sentido } \\
\text { negativo con la satisfacción sexual }\end{array}$ \\
\hline
\end{tabular}

Ratiinrhorn y Viwatwongkasem (2017) encontró esta relación en ambos sexos.

Por otro lado, la actitud favorable al DES dificultó la toma de decisiones en las relaciones sexuales, concretamente la decisión de las mujeres relativa al uso de anticonceptivos se basaba en las preferencias del hombre (Kelly et al., 2017) o en el miedo a ser estigmatizadas (Khumsaen y Gary, 2009). Sin embargo, el trabajo de Fiaer, Pedersen y Sandberg (2015) halló que, en el caso de las mujeres, el DES favorece el control en las relaciones sexuales para promover la seguridad y la higiene. En esta última línea de resultados, se encontró que la actitud a favor del DES se relacionó con una mayor preocupación de las mujeres por el sexo no seguro (Saavedra, Nogueira, y Magalhaes, 2010), la transmisión de ITS (Khumsaen y Gary, 2009) y los embarazos no deseados (Ford y Kittisuksathit, 1994; Saavedra et al., 2010). Se observó que las diferencias que conlleva el DES en la evaluación de los comportamientos sexuales implicó que 
las relaciones sexuales entre dos mujeres se consideren como sexo seguro, ya que tienden a ocurrir en un contexto de relaciones románticas $y$, por tanto, sea innecesario la necesidad de protección (Souto-Pereira, Swainston, y Becker, 2019).

Con respecto a la relación entre apoyo al DES y la propuesta del uso del condón, los resultados no son consistentes. Por un lado, no se encontró relación entre el apoyo a DES y la evaluación de hombres y mujeres en la propuesta de uso de algún método de anticoncepción- barrera, es decir, no se hallaron diferencias en la evaluación sobre quien proponía el uso del condón (Broaddus, Morris, y Bryan, 2010; Penhollow y Young, 2017). En cambio, el estudio de Hynie y Lydon (1995), con una muestra formada exclusivamente por mujeres, sí encontró que las actitudes a favor del DES tradicional implicaban una evaluación más negativa para las mujeres que proponían el uso del condón.

Finalmente, los resultados de dos estudios manifiestan que un mayor apoyo al DES tradicional se relacionó con mayor probabilidad de contraer ITS en mujeres (Bermúdez, Ramiro, Sierra, y Buela-Casal, 2013; Orisaremi, 2017; Teitelman et al., 2013). Un solo estudio encontró esta relación en hombres y mujeres (Bermúdez, Castro, Gude, y Buela- Casal, 2010). Además, la actitud favorable al DES implicó mayor rechazo y estigmatización, para las mujeres que para los hombres, por el diagnóstico de una ITS (Smith, Mysak, y Michael, 2008). Véase la Tabla 2.

\section{DISCUSIÓN}

Se llevó a cabo una revisión sistemática de veintidós artículos científicos que centraron su interés en la relación del doble estándar sexual (DES) con el funcionamiento sexual (FS) y las conductas sexuales de riesgo (CSR), dimensiones fundamentales de la salud sexual, con el objetivo de sintetizar de forma fiable y replicable los conocimientos existentes acerca de esta temática (Perestelo-Pérez, 2013).

En cuanto a las características demográficas de las muestras empleadas en los trabajos revisados, del total de artículos de la revisión, el $31,81 \%$ de los estudios incluyeron una muestra formada exclusivamente por mujeres, un trabajo se realizó con hombres exclusivamente y el resto $(63,63 \%)$ incluía muestras mixtas. El 72,72\% incluyó a adolescentes y/o adultos jóvenes. Al igual que en esta revisión, el metaanálisis de Endendijk, Anneloes, Baar, y Deković (2019) destacó que en la mayoría de las muestras de los estudios del DES se incluyó adultos emergentes, lo que dificulta la generalización de los resultados a la población general (Yanez-Peñúñuri, Martínez-Gómez, y ReyAnacona, 2019). No se han encontrado estudios transculturales, salvo el de Haavio-Mannila y Kontula (2003). Resultaría conveniente contar con muestras más diversas, y hacer comparaciones entre grupos en función del sexo y la edad, así como comparaciones transculturales basadas en medidas invariantes (Sánchez-Fuentes et al., 2020), que permitan aportar información culturalmente sensible (Schmidt, van der Meer, Tydecks, y Bliesener, 2018).

El instrumento estandarizado más empleado para la evaluación del DES ha sido la DSS (Caron et al., 1993), incluido en el 22,7\% de los estudios revisados. En la revisión sobre DES de Bordini y Sperb (2013) también se indica que se trata de una de las escalas más usadas, a pesar de una limitación importante que le caracteriza: no distinguir la percepción de la aceptación social del DES de la aceptación personal. A esta limitación de la DSS, se debe añadir otra propia de la evaluación del DES: la combinación de ítems que evalúan la actitud hacia el DES con otros que evalúan la actitud hacia conductas sexuales en el hombre o en la mujer (Bordini y Sperb, 2013; Sierra et al., 2018). Esto posiblemente genere inconsistencia, pudiendo ser una de las explicaciones a la controversia en los resultados encontrados en las diferentes investigaciones de este campo. Por ello, se ha recomendado el uso de la SDSS (Muehlenhard y Quackenbush, 2011), ya que se compone de ítems paralelos de un mismo comportamiento en el hombre y en la mujer y, además, permite distinguir diferentes tipologías del DES (Endendijk et al., 2019).

Ante estas evidencias, se destaca la necesidad de contar con instrumentos que aseguren la evaluación exclusiva del DES (i.e., que un mismo comportamiento sexual sea valorado en un hombre y en una mujer), y que sean capaces de diferenciar la percepción social de la personal. Además, se requiere la demostración 
Tabla 2

Estudios sobre la relación del doble estándar sexual (DES) y las conductas sexuales de riesgo (CSR)

\begin{tabular}{|c|c|c|c|c|}
\hline Autor/es & $\begin{array}{l}\text { Variables } \\
\text { relacionadas } \\
\text { con el DES }\end{array}$ & Participantes & $\begin{array}{l}\text { Instrumentos } \\
\text { para evaluar } \\
\text { DES }\end{array}$ & Resultados \\
\hline $\begin{array}{l}\text { Bermúdez, Castro, } \\
\text { Gude y Buela-Casal } \\
\text { (2010) }\end{array}$ & ITS & $\begin{array}{c}689 \text { participantes: } 37,8 \% \\
\text { hombres y } 62,2 \% \\
\text { mujeres de } 14 \text { a } 19 \text { años } \\
\text { de edad }(M=17,30)\end{array}$ & $\begin{array}{l}\text { Double Standard } \\
\text { Scale (Caron, } \\
\text { Davis, Halteman y } \\
\text { Stricke, 1993) }\end{array}$ & $\begin{array}{l}\text { El DES predice el riesgo de ITS/VIH a } \\
\text { través de la toma de decisiones en la } \\
\text { pareja }\end{array}$ \\
\hline $\begin{array}{l}\text { Bermúdez, Ramiro, } \\
\text { Sierra y Buela-Casal } \\
\text { (2013) }\end{array}$ & ITS & $\begin{array}{c}599 \text { participantes: } 65,94 \% \\
\text { hombres y } 34,06 \% \text { mujeres } \\
\text { de } 12 \text { a } 18 \text { años de edad } \\
(M \text { hombres }=15,48 ; M \\
\text { mujeres }=15,76)\end{array}$ & $\begin{array}{l}\text { Double Standard } \\
\text { Scale (Caron et al., } \\
\text { 1993) }\end{array}$ & $\begin{array}{l}\text { La baja adherencia al DES se } \\
\text { relaciona de forma indirecta, } \\
\text { mediante el poder de la relación, con } \\
\text { el menor riesgo de infección por VIH } \\
\text { en las mujeres }\end{array}$ \\
\hline $\begin{array}{l}\text { Broaddus Morris y } \\
\text { Bryan (2010) }\end{array}$ & $\begin{array}{l}\text { Propuesta del } \\
\text { uso método } \\
\text { barrera- } \\
\text { anticoncepción }\end{array}$ & $\begin{array}{c}150 \text { participantes: } 54 \% \\
\text { hombres y } 46 \% \text { mujeres de } \\
18 \text { a } 24 \text { años de edad ( } M \\
=19 \text { ) }\end{array}$ & $\begin{array}{c}\text { Cuestionario ad } \\
\text { hoc basado en } \\
\text { Bryan, Aiken y West } \\
\text { (1999) }\end{array}$ & $\begin{array}{l}\text { EI DES no se asocia con la propuesta } \\
\text { del uso del condón en una relación } \\
\text { sexual }\end{array}$ \\
\hline Danube et al. (2015) & $\begin{array}{l}\text { Uso método } \\
\text { barrera- } \\
\text { anticoncepción }\end{array}$ & $\begin{array}{l}364 \text { mujeres de } 21 \text { a } 35 \\
\text { años de edad }(M=25)\end{array}$ & $\begin{array}{l}\text { Double Standard } \\
\text { Scale (DSS; Caron } \\
\quad \text { et al., 1998) }\end{array}$ & $\begin{array}{l}\text { La aprobación del DES se relaciona } \\
\text { de forma indirecta con la intención de } \\
\text { mantener sexo sin protección }\end{array}$ \\
\hline $\begin{array}{l}\text { Fjaer, Pedersen y } \\
\text { Sandberg (2015) }\end{array}$ & $\begin{array}{l}\text { Preocupación } \\
\text { por mantener } \\
\text { sexo no seguro }\end{array}$ & $\begin{array}{c}41 \text { participantes: } 39,02 \% \\
\text { hombres y } 67,98 \% \\
\text { mujeres de } 18 \text { a } 19 \text { años } \\
\text { de edad* }\end{array}$ & Entrevista & $\begin{array}{l}\text { EI DES está presente en el discurso de } \\
\text { las mujeres jóvenes, indicando que } \\
\text { en sus experiencias sexuales deberían } \\
\text { ejercer el autocontrol para promover } \\
\text { la seguridad, higiene y autonomía }\end{array}$ \\
\hline $\begin{array}{l}\text { Ford y Kittisuksathit } \\
\qquad(1994)\end{array}$ & $\begin{array}{l}\text { Preocupación } \\
\text { por mantener } \\
\text { sexo no seguro }\end{array}$ & $\begin{array}{l}\text { Hombres y mujeres de } 15 \text { a } \\
24 \text { años de edad* }\end{array}$ & Discusión/Entrevista & $\begin{array}{c}\text { EI DES hace que los hombres tiendan a } \\
\text { discutir los inconvenientes de las ITS y } \\
\text { las mujeres los embarazos no } \\
\text { deseados }\end{array}$ \\
\hline Hynie y Lydon (1995) & $\begin{array}{l}\text { Propuesta del } \\
\text { uso de método } \\
\text { barrera- } \\
\text { anticoncepción }\end{array}$ & $\begin{array}{l}57 \text { mujeres de } 17 \text { a } 30 \\
\text { años de edad }(M=20)\end{array}$ & $\begin{array}{l}\text { Cuestionarios ad } \\
\text { hoc }\end{array}$ & $\begin{array}{l}\text { EI DES favorece que las mujeres que } \\
\text { proponen el uso del condón en una } \\
\text { relación sexual sean evaluadas de } \\
\text { forma negativa }\end{array}$ \\
\hline Kelly et al. (2017) & $\begin{array}{l}\text { Uso método } \\
\text { barrera- } \\
\text { anticoncepción }\end{array}$ & $\begin{array}{l}94 \text { mujeres } \\
\text { de } 16 \text { a } 49 \text { años de edad* }\end{array}$ & Entrevista & $\begin{array}{c}\text { El DES impregna las prácticas sexuales } \\
\text { y anticonceptivas de las mujeres, } \\
\text { centrándolas en el placer y el } \\
\text { deseo de los hombres, y otorgando } \\
\text { poca importancia a la experiencia } \\
\text { orgásmica }\end{array}$ \\
\hline $\begin{array}{c}\text { Khumsaen y Gary } \\
\text { (2009) }\end{array}$ & $\begin{array}{l}\text { Uso método } \\
\text { barrera- } \\
\text { anticoncepción }\end{array}$ & $\begin{array}{l}270 \text { participantes: } 64,4 \% \\
\text { hombres y } 35,6 \% \\
\text { mujeres de } 18-21 \text { años de } \\
\text { edad }(M=19,18)\end{array}$ & Preguntas ad hoc & $\begin{array}{l}\text { La toma de decisiones de las mujeres } \\
\text { se dificulta por la presencia de } \\
\text { DES, con menos énfasis en el uso } \\
\text { de anticonceptivos por miedo a ser } \\
\text { estigmatizadas como sexualmente } \\
\text { experimentadas. Las mujeres tienen } \\
\text { el doble de probabilidades de } \\
\text { mencionar las ITS que los hombres }\end{array}$ \\
\hline $\begin{array}{l}\text { Lotfi, Tehrani, Khoei, } \\
\text { Yaghmaei y Dworkin, } \\
\text { (2013) }\end{array}$ & $\begin{array}{l}\text { Uso método } \\
\text { barrera- } \\
\text { anticoncepción }\end{array}$ & $\begin{array}{c}22 \text { mujeres } \\
\text { de } 21 \text { a } 49 \text { años de edad, } \\
\text { dividas en tres grupos de } \\
\text { edad: } \\
21-29 \text { años }(22,7 \%), 30-39 \\
\text { años }(45,5 \%) \text { y } 40-49 \text { años } \\
(31,8 \%)^{*}\end{array}$ & Entrevista & $\begin{array}{l}\text { Las normas de género, entre las que } \\
\text { se encuentra el DES, son percibidas } \\
\text { como una barrera fundamental para } \\
\text { la protección contra el riesgo }\end{array}$ \\
\hline Orisaremi (2017) & ITS & $\begin{array}{c}53 \text { participantes: } 50,95 \% \\
\text { hombres y } 49,05 \% \\
\text { mujeres mayores de } 15 \\
\text { años* }\end{array}$ & Discusión/Entrevista & $\begin{array}{l}\text { Las prácticas sociales y culturales } \\
\text { relacionadas con el género, como } \\
\text { el DES, facilitan la transmisión del VIH } \\
\text { en mujeres casadas. Las mujeres con } \\
\text { relaciones heterosexuales mantenían } \\
\text { una posición subordinada que } \\
\text { aumenta su vulnerabilidad a contraer } \\
\text { ITS, incluido el VIH }\end{array}$ \\
\hline
\end{tabular}


Tabla 2 (Continuación)

Estudios sobre la relación del doble estándar sexual (DES) y las conductas sexuales de riesgo (CSR)

\begin{tabular}{|c|c|c|c|c|}
\hline Autor/es & $\begin{array}{l}\text { Variables } \\
\text { relacionadas } \\
\text { con el DES }\end{array}$ & Participantes & $\begin{array}{l}\text { Instrumentos } \\
\text { para evaluar } \\
\text { DES }\end{array}$ & Resultados \\
\hline $\begin{array}{c}\text { Penhollow y Young } \\
\text { (2017) }\end{array}$ & $\begin{array}{l}\text { Propuesta del } \\
\text { uso de método } \\
\text { barrera- } \\
\text { anticoncepción }\end{array}$ & $\begin{array}{c}574 \text { participantes: } 36 \% \\
\text { hombres y } 64 \% \text { mujeres } \\
\text { menores de } 25 \text { años* }\end{array}$ & $\begin{array}{l}\text { Cuestionario ad } \\
\text { hoc. }\end{array}$ & $\begin{array}{l}\text { No hay relación del DES en la } \\
\text { propuesta del uso del condón en una } \\
\text { relación sexual }\end{array}$ \\
\hline $\begin{array}{l}\text { Saavedra, Nogueira y } \\
\text { Magalhaes (2010) }\end{array}$ & $\begin{array}{l}\text { Preocupación } \\
\text { por mantener } \\
\text { sexo no seguro }\end{array}$ & $\begin{array}{c}90 \text { participantes de } 15 \text { a } \\
19 \text { años: } 37,78 \% \\
\text { hombres y } 62,22 \% \\
\text { mujeres, }(M=17,07)\end{array}$ & Preguntas ad hoc. & $\begin{array}{l}\text { Ambos sexos admiten la existencia de } \\
\text { DES, pero solo las mujeres incluyen } \\
\text { en su discurso el sexo no seguro, } \\
\text { prestando mayor atención a la } \\
\text { evitación del embarazo }\end{array}$ \\
\hline $\begin{array}{l}\text { Smith, Mysak y } \\
\text { Michael (2008) }\end{array}$ & ITS & $\begin{array}{l}151 \text { participantes: } 49,67 \% \\
\text { hombres y } 50,33 \% \\
\text { mujeres }(M=18)^{\star}\end{array}$ & $\begin{array}{l}\text { Cuestionario ad } \\
\text { hoc }\end{array}$ & $\begin{array}{l}\text { La presencia del DES se relacionó } \\
\text { con mayor rechazo y estigmatización } \\
\text { de las mujeres que padecían ITS en } \\
\text { comparación con los hombres con } \\
\text { ITS }\end{array}$ \\
\hline $\begin{array}{l}\text { Srijaiwong, Sinshu, } \\
\text { Ratinrhorn y } \\
\text { Viwatwongkasem } \\
\text { (2017) }\end{array}$ & $\begin{array}{l}\text { Uso método } \\
\text { barrera- } \\
\text { anticoncepción }\end{array}$ & $\begin{array}{l}3.192 \text { participantes: } 36,1 \% \\
\text { hombres y } 63,9 \% \\
\text { mujeres de } 15 \text { a } 19 \text { años } \\
(M=16,5)\end{array}$ & $\begin{array}{l}\text { Sexual Double } \\
\text { Standard Scale } \\
\text { (SDSS; Muehlenhard } \\
\text { y Quackenbush, } \\
\text { 2011). }\end{array}$ & EI DES predice las CSR \\
\hline $\begin{array}{c}\text { Souto Pereira, } \\
\text { Swainston y Becker } \\
\text { (2019) }\end{array}$ & $\begin{array}{l}\text { Uso método } \\
\text { barrera- } \\
\text { anticoncepción }\end{array}$ & $\begin{array}{c}17 \text { mujeres } \\
\text { de } 20 \text { a } 58 \text { años de edad } \\
(M=35)\end{array}$ & Entrevista & $\begin{array}{l}\text { Aunque cada participante tenía } \\
\text { conocimiento de la posibilidad de } \\
\text { enfermedades de transmisión sexual, } \\
\text { el DES favorece considerar el sexo } \\
\text { entre dos mujeres como "seguro" e } \\
\text { innecesario el uso de métodos de } \\
\text { protección }\end{array}$ \\
\hline $\begin{array}{l}\text { Teitelman, Tennille, } \\
\text { Bohinski, Jemmott y } \\
\text { Jemmott (2013) }\end{array}$ & $\begin{array}{l}\text { Uso método } \\
\text { barrera- } \\
\text { anticoncepción. } \\
\text { ITS }\end{array}$ & $\begin{array}{l}64 \text { mujeres de 14-17 } \\
\text { años de edad }(M=16)\end{array}$ & Entrevista & $\begin{array}{l}\text { La presencia de normas de género } \\
\text { comunes al DES favorece que } \\
\text { las mujeres puedan estar más } \\
\text { predispuestas a renunciar a prácticas } \\
\text { de sexo seguro. Las mujeres con } \\
\text { pareja son más vulnerables al } \\
\text { riesgo de contagio de VIH/ITS. Los } \\
\text { hombres con apoyo a DES tienen más } \\
\text { probabilidad de mantener relaciones } \\
\text { sexuales sin protección }\end{array}$ \\
\hline
\end{tabular}

de la invarianza factorial y métrica a través de los grupos poblacionales objeto de comparación, para garantizar la exactitud y la equidad de la medida (Muñiz y Fonseca-Pedrero, 2019). De este modo, sería posible estudiar el DES con muestras más diversas haciendo comparaciones entre colectivos distintos y estableciendo de forma rigurosa la influencia de variables socioculturales.

A pesar de haber escasos estudios que aborden la relación entre el DES y el FS, parece ser que el DES afecta negativamente a las dimensiones del FS. Cabe subrayar que no se han encontrado estudios que relacionasen el DES con la excitación sexual. La excitación sexual como rasgo y como componente de la función sexual influye en la autovaloración de la capacidad sexual (Granados, Salinas, y Sierra, 2017, 2018; Sierra, López-Herrera, Álvarez- Muelas, Arcos-Romero, y Calvillo, 2018), y tiene un peso importante en la satisfacción sexual de hombres y mujeres (Sánchez-Fuentes, Moyano, Granados, y Sierra, 2019; Sánchez- Fuentes, Salinas, y Sierra, 2016; Sierra, Vallejo-Medina, Granados, Moyano, y Sánchez- Fuentes, 2015), por lo que futuros estudios deberían abordar esta relación. La mayoría de los estudios se centran en la relación del DES con la satisfacción sexual, pero sin llegar a resultados concluyentes. Esta inconsistencia en los resultados obtenidos podría deberse a varias razones. Una de ellas podría ser el uso de instrumentos para evaluar la satisfacción sexual no fundamentados en modelos teóricos sólidos, pues 
tal como señalan Sánchez- Fuentes et al. (2014), en ocasiones se han utilizado preguntas ad hoc o escalas que confunden satisfacción sexual con factores asociados a la misma (e.g., actitudes sexuales, deseo o excitación sexual). Resultaría conveniente, por tanto, contar con instrumentos de evaluación de la satisfacción sexual basados en modelos teóricos consolidados, como es el caso del Interpersonal Exchange Model of Sexual Satisfaction Questionnaire (IEMSSQ; Lawrance, Byers, y Cohen, 2011 ; véase Calvillo, SánchezFuentes, Parrón Carreño, y Sierra, 2019; SánchezFuentes, Santos-Iglesias, Byers, y Sierra, 2015). Otra razón podría ser el hecho de no evaluar de manera independiente el apoyo personal al DES, y la percepción que tiene la persona encuestada del apoyo que la sociedad concede al DES (véase Gómez Berrocal, Vallejo-Medina, Moyano, y Sierra, 2019). Las discrepancias entre la actitud personal y la percepción de la norma social pueden generar incomodidad psicológica y ambivalencia (Dovidio y Gaentner, 2004; Gaertner, Sedikides, Vevea, y Luzzini, 2002). Por lo tanto, sin la diferenciación de ambas medidas se imposibilita conocer la influencia de la discrepancia entre ellas sobre la satisfacción sexual. Finalmente, dado que la excitación sexual constituye un factor importante en la explicación de la satisfacción sexual (Sánchez-Fuentes et al., 2014 , 2016, 2019), cabe pensar que la relación del DES con la satisfacción sexual pudiera estar mediada por la propensión a excitarse/inhibirse sexualmente planteada por el Modelo de Control Dual de la respuesta sexual (Bancroft y Janssen, 2000; Janssen y Bancroft, 2007).

En cuanto a la relación del DES con CSR, se observa un incremento del número de estudios en los últimos cinco años. Los resultados encontrados confirman que la actitud a favor del DES favorece las conductas sexuales de riesgo. El apoyo al DES predice un menor uso de protección (métodos barrera-anticoncepción) en cuatro estudios (Danube et al., 2015; Lotfi et al., 2013; Srijaiwong et al., 2017; Teitelman et al., 2013) y mayor probabilidad de contraer ITS en otros cuatro (Bermúdez et al., 2010, 2013; Orisaremi, 2017; Teitelman et al., 2013). Cabe destacar que el DES resulta más perjudicial para las mujeres que para los hombres. La mayoría de los estudios encuentran una asociación positiva entre DES y las CSR en las mujeres (Danube et al., 2015; Lotfi et al., 2013; Teitelman et al., 2013), apreciándose una mayor probabilidad de contraer ITS (Bermúdez et al., 2013; Orisaremi, 2017; Teitelman et al., 2013) y una mayor dificultad en la toma de decisiones en las relaciones sexuales (Kelly et al., 2017; Khumsaen y Gary, 2009). Resulta conveniente resaltar algunas controversias observadas entre los estudios, no pudiendo llegar a resultados concluyentes sobre la influencia del DES en aspectos como la preocupación por mantener sexo no seguro o la iniciativa del uso del condón. De este modo, se enfatiza la necesidad de más investigación en conductas sexuales de riesgo teniendo en cuenta el papel directo que puede desempeñar el DES. Tal como indican Ramiro-Sánchez, Ramiro, Bermúdez y Buela-Casal (2018), se recomienda incluir la intervención y/o prevención específica de las creencias sexistas en programas de prevención de ITS/NIH.

En definitiva, se destaca el papel que el doble estándar sexual tiene en el estudio de la salud sexual, específicamente en el funcionamiento sexual y las conductas sexuales de riesgo. Se concluye que la actitud a favor del DES resulta perjudicial para el funcionamiento sexual, en concreto para el deseo sexual, la experiencia orgásmica y la satisfacción sexual. En cambio, no se encuentran estudios que relacionen el DES con la excitación sexual. A su vez, el apoyo al DES favorece las conductas sexuales de riesgo en mayor medida en las mujeres. Se plantea la necesidad de más investigación para ampliar el conocimiento del DES de cara a integrarlo en programas de salud sexual. Se destaca entre las limitaciones del estudio la imposibilidad de llevar a cabo metaanálisis debido a la heterogeneidad de las muestras de los trabajos incluidos en la revisión, de la evaluación de los constructos y de los resultados encontrados.

\section{- Conflicto de intereses.}

Los autores declaran no tener ningún conflicto de intereses.

\section{- Agradecimientos}

Este trabajo ha sido financiado por el Ministerio de Economía y Competitividad a través del Proyecto de Investigación PSI201458035-R y de la Beca FPU 16/04429 para la Formación de Profesorado Universitario, formando parte de la Tesis Doctoral de la primera autora (Programa de Doctorado en Psicología B13 561 ; RD 99/2011). 


\section{REFERENCIAS}

*Referencia incluida en la revisión sistemática

Álvarez-Muelas, A., Gómez-Berrocal, C., VallejoMedina, P., y Sierra, J. C. (2019). Invariance of Spanish version of Sexual Double Standard Scale across sex, age, and education level. Psicothema, 31, 465-474. https://dx.doi. org/10.7334/psicothema2019.102

Arcos-Romero, A. I., ySierra, J. C. (2018). Revisión sistemática sobre la experiencia subjetiva del orgasmo [Systematic review of the subjective experience of orgasm]. Revista Internacional de Andrología, 16, 75-81. https://dx.doi. org/10.1016/i.androl.2017.09.003

Bancroft, J., y Janssen, E. (2000). The dual control model of male sexual response: $A$ theoretical approach to centrally mediated erectile dysfunction. Neuroscience and Biobehavioral Reviews, 24, 571-579.

*Bermúdez, M. P., Castro, A., Gude, F., y BuelaCasal, G. (2010). Relationship power in the couple and sexual double standard as predictors of the risk of sexually transmitted infections and HIV: Multicultural and gender differences. Current HIV Research, 8, 172-178. https:// dx.doi.org/10.2174/157016210790442669

*Bermúdez, M. P., Ramiro, M. T., Sierra, J. C., y Buela-Casal, G. (2013). Construcción de un índice de riesgo para la infección por el $\mathrm{VIH}$ y su relación con la doble moral y el poder diádico en adolescentes [Construction of a risk index of HIV infection and its relation to double morale and dyadic power in adolescents ]. Anales de Psicología, 29, 917-922. https:// dx.doi.org/10.6018/analesps.29.3.136931

Bordini, G. S, y Sperb, T. M. (2013). Sexual double standard: A review of the literature between 2001 and 2010. Sexuality y Culture, 17, 686-704. https://dx.doi.org/10.1007/ s12119-012-9163-0

*Broaddus, M. R., Morris, H., y Bryan, A. D. (2010). "It's not what you said, it's how you said it: Perceptions of condom proposers by gender and strategy. Sex Roles, 62, 603- 614. https://dx.doi.org/10.1007/s $11199-009$ 9728-z
Bryan, A. D., Aiken, L. S., y West, S. G. (1999). The impact of males proposing condom use on perceptions of an initial sexual encounter. Personality and Social Psychology Bulletin, 25, 275-286. https://dx.doi. org/10.1177\%2F0146167299025003001

Calvillo, C., Sánchez-Fuentes, M. M., Parrón Carreño, T., y Sierra, J. C. (2019). Validation of the Interpersonal Exchange Model of Sexual Satisfaction Questionnaire in adults with same-sex partner. International Journal of Clinical and Health Psychology. Publicación en avance en línea. https://dx.doi. org/10.1016/i.ijchp.2019.07.005

Calvillo, C., Sánchez-Fuentes, M. M., y Sierra, J. C. (2018). Revisión sistemática sobre la satisfacción sexual en parejas del mismo sexo [Systematic review on sexual satisfaction in same-sex couples]. Revista Iberoamericana de Psicología y Salud, 9, 115-136. https:// dx.doi.org/10.23923/i.rips.2018.02.018

Caron, S. L., Davis, C.M., Halteman, W. A., yStickle, M. (1993). Predictors of condom-Publicación en avance en línea. Related behaviors among first-year college students. The Journal of Sex Research, 30, 252-259. https://dx.doi. org/10.1080/00224499309551709

Caron, S. L., Davis, C. M., Halteman, W. A., y Stickle, M. (1998). Double Standard Scale. En C. M. Davis, W. L. Yarber, R. Bauserman, G. Schreer, y S. L. Davis (Eds.), Handbook of sexuality-related measures (pp. 182-183). Thousand Oaks, CA: Sage.

Carrobles, J. A., y Sanz, A. (1991). Terapia sexual [Sexual Therapy]. Madrid, Spain: Fundación Universidad Empresa (UNED).

*Danube, C. L., Norris, J., Stappenbeck, C. A., Davis, K. C., George, W. H., Zawacki, T., Abdallah, D. A. (2015). Partner type, sexual double standard endorsement, and ambivalence predict abdication and unprotected sex intentions in a community sample of young women. The Journal of Sex Research, 53, 601-613. https://dx.doi.org/1 $0.1080 / 00224499.2015 .1061631$

Dovidio, J. F., y Gaertner, S. L. (2004). Aversive racism. En M. P. Zanna (Ed.), Advances in experimental social psychology (pp. 1-51). 
San Diego, CA: Academic Press.

Endendiik, J. J., van Baar, A. L., y Deković, M. (2019). He is a stud, she is a slut! A metaanalysis on the continued existence of sexual double standards. Personality and Social Psychology Review, 24, 163-190. https:// dx.doi.org/10.1177/1088868319891310

Fariña, F., Arce, R., y Novo, M. (2002). Heurístico de anclaje en las decisiones judiciales [Anchorage in judicial decision making]. Psicothema, 14, 39-46.

*Fjaer, E. G., Pedersen, W., y Sandberg, S. (2015). I'm not one of those girls. Gender \& Society, 29, 960-981 https://dx.doi. $\mathrm{org} / 10.1177 / 0891243215602107$

*Ford, N. J., y Kittisuksathit, S. (1994). Destinations and changing nature of the sexual expressions of Thai young. Aids Care, 6, 517-531. https:// dx.doi.org/10.1080/09540129408258667

Gaertner, L., Sedikides, C., Vevea, J. L., y Luzzini, J. (2002). The "I," the "we," and the "when": A meta-analysis of motivational primacy in selfdefinition. Journal of Personality and Social Psychology, 83, 574-591 https://dx.doi. org/10.1037//0022-3514.83.3.574

Gómez Berrocal, M. C., Vallejo-Medina, P., Moyano, N., y Sierra, J. C. (2019). Sexual double standard: A psychometric study from a macropsychological perspective among the Spanish heterosexual population. Frontiers in Psychology, 10, 1869. https://dx.doi. org/10.3389/fpsyg.2019.01869

Granados, M. R., Salinas, J. M., y Sierra, J. C. (2017).Spanish version ofthe Sexual Excitation/ Sexual Inhibition Inventory for women: Factorial structure, reliability and validitiy evidence. International Journal of Clinical and Health Psychology, 17, 46-55. https://dx.doi. org/10.1016/i.iichp.2016.09.003

Granados, M. R., Salinas, J. M., y Sierra, J. C. (2018). Psychometric properties of the Spanish version of the Sexual Inhibition/ Sexual Excitation Scales for men. Archives of Sexual Behavior, 47, 783-796. https://dx.doi. org/10.1007/s10508-017-0992-7

Guo, Y. (2019). Sexual double standards in white and Asian Americans: Ethnicity, gender, and acculturation. Sexuality \& Culture, 23,
57-95. https://dx.doi.org/10.1007/s12119018-9543-1

Gutiérrez-Quintanilla, J. R., Rojas-García, A., y Sierra, J. C. (2010). Comparación transcultural de la doble moral sexual entre estudiantes universitarios salvadoreños y españoles [Cross-cultural comparison of the sexual double standard between Salvadoran and Spanish college students]. Revista Salvadoreña de Psicología, 1, 31 - 51.

*Haavio-Mannila, E., y Kontula, O. (2003). Single and Double Sexual Standards in Finland, Estonia and St. Petersburg. The Journal of Sex Research, 40, 36-49. https:// dx.doi.org/10.1080/00224490309552165

Harrison, A., O'Sullivan, L. F., Hoffman, S., Dolezal, C., y Morrell, R. (2006). Gender role and relationships norms among young adults in South Africa: Measuring the context of masculinity and HIV risk. Journal of Urban Health, 83, 709-722. https://dx.doi. org/10.1007/s $11524-006-9077-y$

*Horne, S., y Zimmer-Gembeck, M. J. (2006). The female sexual subjectivity inventory: Development and validation of a multidimensional inventory for late adolescents and emerging adults. Psychology of Women Quarterly, 30, 125-138. https://dx.doi. org/10.1111/i.1471-6402.2006.00276.x

*Hynie, M., y Lydon, J. E. (1995). Women's perceptions of female contraceptive behavior: Experimental evidence of sexual double standard. Psychology of Women Quarterly, 19, 563-581. https://dx.doi. org/10.1111/i.1471-6402.1995.tb00093.x

Isorna Folgar, M., Fariña Rivera, F., Sierra, J. C., y Vallejo-Medina. P. (2015). Binge drinking: conductas sexuales de riesgo y facilitadoras del asalto sexual. Suma Psicológica, 22, 1-8. https://dx.doi.org/10.1016/i. sumpsi.2015.05.001

*Jackson, S. M., y Cram, F. (2003). Disrupting the sexual double standard: Young women's talk about heterosexuality. British Journal of Social Psychology, 42, 113-127. https://dx.doi. $\mathrm{org} / 10.1348 / 014466603763276153$

Janssen, E., y Bancroft, J. (2007). The Dual Control Model: The role of sexual inhibition 
and excitation in sexual arousal and behavior. En E. Janssen (Ed.), The psychophysiology of sex (pp. 197-222). Bloomington, IN: Indiana University Press.

*Kelly, M., Inoue, K., Barrat, A., Bateson, D., Rutherford, A., y Ritchers, J. (2017). Performing (heterosexual) femininity: Female agency and role in sexual life and contraceptive use-a qualitative study in Australia. Culture, Health \& Sexuality, 19, 240-255. https://dx.doi.org/10. 1080/13691058.2016.1214872

*Khumsaen, N., y Gary, F. A. (2009). Determinants of actual condom use among adolescents in Thailand. Journal of the Association of Nurses in AIDS Care, 20, 218-229. https://dx.doi. org/10.1016/i.jana.2008.12.006

Lawrance, K., Byers, E. S., y Cohen, J. N. (2011). Interpersonal Exchange Model of Sexual Satisfaction Questionnaire. En T. D. Fisher, C. M. Davis, W. L. Yarber y S. L. Davis (Eds.), Handbook of sexuality-related measures $\left(3^{a}\right.$ ed. pp. 525-530). Nueva York, NY: Routledge. Liberati, A., Altman, D. G., Tetzlaff, J, Mulrow, Gøtzsche, P. C., loannidis J. P., ...Moher, D. (2010). The PRISMA statement for reporting systematic reviews and meta-analyses of studies that evaluate health care interventions: Explanation and elaboration. PLoSMedicine, 6, e1000100. https://dx.doi.org/10.1371/ journal.pmed.1000100

*Lotfi, R., Tehrani, F. R., Khoei, E. M., Yaghmaei, F., y Dworkin, S. L. (2013). How do women at risk of HIV/AIDS in Iran perceive gender norms and gendered power relations in the context of safe sex negotiations? Archives of Sexual Behavior, 42, 873-881. https://dx.doi.org/10.1007/ s10508-012-0040-6

Lottes, I. (2000). New researchs on sexual health. En I. Lottes y $\mathrm{O}$. Kontula (Eds.), New views on sexual health: The case of Finland (pp. 7-28). Helsinki, Finland: Population Research Institute. *Marques, N. M., Viera, R. X., y Pechorro, P. S. (2013). The sexual double standard in a masculine way: A Portuguese transgenerational perspective. Revista Internacional de Andrología, 11, 138-143. https://dx.doi. org/10.1016/i.androl.2013.06.001

Milhausen, R. R., y Herold, E. S. (2001).
Reconceptualizing the sexual double standard. Journal of Psychology and Human Sexuality, 13, 63-83. https://dx.doi.org/10.1300/ J056v13n02 05

Monge, F. S., Sierra, J. C., y Salinas, J. M. (2013). Equivalencia factorial y métrica de la Double Standard Scale por sexo y edad [Factorial and metric equivalence of Double Standard Scale by gender and age]. Suma Psicológica, 20, 7-14.

Monteiro, A., Vázquez, M.J., Seijo, D., y Arce, R. (2018). ¿̇Son los criterios de realidad válidos para clasificar y discernir entre memorias de hechos auto-experimentados y de eventos vistos en vídeo? [Are the reality criteria valid to classify and to discriminate between memories of self-experienced events and memories of video-observed events?]. Revista Iberoamericana de Psicología y Salud, 9, 149-160. https://doi.org/10.23923/i. rips.2018.02.020

Morales-Toro, V., Guillén-Riquelme, A., y Quevedo-Blasco, R. (2019). Maltrato infantily trastornos mentales en delincuente juveniles: una revisión sistemática [Child abuse and mental disorders in juvenile delinquents: A systematic review]. Revista de Investigación en Educación, 17, 218-238.

Muehlenhard, C. L., y Quackenbush, D. M. (2011). The sexual double standard scale. En T. D. Fisher, C. M. Davis, W. L. Yarber, R. Bauserman, G. E. Schree y S. L. David (Eds.), Handbook of sexuality-related measures (2a ed., pp.199-200). Philadelphia, MA: Taylor \& Francis.

Muñiz, J., y Fonseca-Pedrero. (2019). Diez pasos para la construcción de un test [Ten steps fo test development]. Psicothema, 31, 7-16. https:// dx.doi.org/10.7334/psicothema2018.291

National Heart, Lung, and Blood Institute. (2014). Quality assessment tool for observational cohort and cross-sectional studies. Recuperado de https://www.nhlbi. nih.gov/health-pro/guidelines/in-develop/ cardiovascular-risk-reduction/tools/cohort

*Orisaremi, T. C. (2017). Gender relations and HIV transmission in north-central Nigeria. Sexuality \& Culture, 21, 991-1017. https:// 
dx.doi.org/10.1007/s12119-017-9433-y

Pawlowsky-Glahn, V., Egozcue, J. J., y PlanesPedra, M. (2018). Survey data on perceptions of contraceptive methods as compositional tables. Revista Latinoamericana de Psicología, 50, 179-186. https://dx.doi.org/10.14349/ rlp.2018.v50.n3.5

*Penhollow, T. M., y Young, M. (2017). Alcohol use, hooking- up, condom use: Is there a sexual double standard? American Journal of Health Behavior, 41, 92-103. https://dx.doi. org/10.5993/AJHB.41.1.10

Perestelo-Pérez, L. (2013). Standards on how to develop and report systematic reviews in psychology and health. International Journal of Clinical and Health Psychology, 13, 4957. https://dx.doi.org/10.1016/S16972600(13)70007-3

Ramiro-Sánchez, T., Ramiro, M. T., Bermúdez, M. P., y Buela-Casal, G. (2018). Sexism and sexual risk behavior in adolescents: Gender differences. International Journal of Clinical and Health Psychology, 18, 245-253. https:// dx.doi.org/10.1016/i.ijchp.2018.04.002

*Saavedra, L., Nogueira, C., y Magalhaes, S. (2010). Discourses of Portuguese adolescents about sexuality and love: Implications for sexual education. Educação \& Sociedade, 31, 135-156. https://dx.doi.org/10.1590/ S0101-73302010000100008

Sakaluk, J. K., y Milhausen, R. R. (2012). Factors influencing university students' explicit and implicit sexual double standards. Journal of Sex Research, 49, 464-476. https://dx.doi.or $\mathrm{g} / 10.1080 / 00224499.2011 .569976$

Sánchez-Fuentes, M. M., Moyano, N., GómezBerrocal, C., y Sierra, J. C. (2020). Invariance of the Sexual Double Standard Scale: A cross-cultural study. International Journal of Environmental Research and Public Health, 17, 1569. https://dx.doi.org/10.3390/ ijerph 17051569

Sánchez-Fuentes, M. M., Moyano, N., Granados, R., y Sierra, J. C. (2019). Validation of the Spanish version of the Arizona Sexual Experience Scale (ASEX) using self-reported and psychophysiological measures. Revista Iberoamericana de Psicología y Salud,
10, 1-14. https://dx.doi.org/10.23923/i. rips.2019.01.021

Sánchez-Fuentes, M. M., Salinas, J. M., y Sierra, J. C. (2016). Use of an ecological model to study sexual satisfaction in a heterosexual Spanish sample. Archives of Sexual Behavior, 45, 1973-1988. https://dx.doi.org/10.1007/ s10508-016-0703-9

Sánchez-Fuentes, M. M., Santos-Iglesias, P., Byers, S., y Sierra, J. C. (2015). Validation of the Interpersonal Exchange Model of Sexual Satistaction Questionnaire in a Spanish sample. The Journal of Sex Research, 52, 1028-1041. https://dx.doi.org/10.1080/00 224499.2014 .989307

Sánchez-Fuentes, M. M., Santos-Iglesias, P., y Sierra, J. C. (2014). A systematic review of sexual satisfaction. International Journal of Clinical and Health Psychology, 14, 6775. https://dx.doi.org/10.1016/S1697. 2600(14)70038-9

*Santos-Iglesias, P., Sierra, J. C., García, M., Martínez, A. Sánchez, A., y Tapia, M. I. (2009). Índice de Satisfacción Sexual (ISS): un estudio sobre fiabilidad y validez [Index of Sexual Satisfaction (ISS): A study on the reliability and validity]. International Journal of Psychology and Psychological Therapy, 9, 259-273. https://dx.doi.org/10.1016/ S0120-0534(14)70016-0

Schmidt, S., van der Meer, E., Tydecks, S., y Bliesener, T. (2018). How culture and migration affect risk assessment. European Journal of Psychology Applied to Legal Context, 10, 65-78. https://dx.doi.org/10.5093/ ejpalc2018a7

Senn, T. E., Carey, M. P., Vanable, P. A., CouryDoniger, P., y Urban, M. (2007). Characteristics of sexual abuse in childhood and adolescence influence sexual risk behavior in adulthood. Archives of Sexual Behavior, 36, 637-645. https://dx.doi.org/10.1007/s1058-0069109-4

*Smith, G., Mysak, K., y Michael, S. (2008). Sexual double standards and sexuallity trasmitted illnesses: Social rejection and stigmatization of women. Sex Roles, 58, 391 401. https://dx.doi.org/10.1007/s 11199 - 


\section{7-9339-5}

Sierra, J. C., López-Herrera, F., Álvarez-Muelas, A., Arcos-Romero, A. I., y Calvillo, C. (2018). La autoestima sexual: su relación con la excitación sexual [The sexual self- esteem: Its relationship with the sexual arousal]. Suma Psicológica, 25, 146-152. https://dx.doi. org/10.14349/sumapsi.2018.v25.n2.6

Sierra, J. C., Monge, F. S., Santos-Iglesias, P., Rodriguez, K., y Aparicio, D. L. (2010). Propiedades psicométricas de las versiones en español de la Double Standard Scale (DSS) y de la Rape Supportive Scale (RSAS) en mujeres peruanas [Psychometric properties of Spanish version of Double Standard Scale (DSS) and of Rape Supportive Attitude Scale (RSAS) in Peruvian women]. Cuadernos de Medicina Psicosomática y Psiquiatría de Enlace, 95, 57 66.

Sierra, J. C., Moyano, N., Vallejo-Medina, P., y Gómez- Berrocal, C. (2018). An abridged Spanish version of Sexual Double Standard Scale: Factorial structure, reliability and validity evidence. International Journal of Clinical and Health Psychology, 18, 69-80. https://dx.doi. org/10.1016/i.iichp.2017.05.003

Sierra, J. C., Vallejo-Medina, P., Granados, R., Moyano, N., y Sánchez-Fuentes, M. M. (2015, julio). Relación de la satisfacción sexual con la respuesta sexual en función del sexo y edad [Relationship of sexual satisfaction with sexual response by sex and age]. Ponencia presentada en el XXXV Congreso Interamericano de Psicología, Lima, Perú.

*Souto-Pereira, S., Swainston, K., y Becker, S. (2019). The discursive construction of lowrisk to sexually transmitted diseases between women who are sexually active with women. Culture, Health \& Sexualiry, 1 1, 1-13. https:// dx.doi.org/10.1080/13691058.2018.1554 188

Sprecher, S. (1989). Premarital sexual standards for different categories of individuals. Journal of Sex Research, 26, 232-248.

*Srijaiwong, S., Sinshu, S., Ratinrhorn, A., y Viwatwongkasem, C. (2017). Factors influencing sexual behaviors among Thai adolescents. Journal of Population and
Social Studies, 25, 171-193. https://dx.doi. org/10.25133/JPSSn3.002

*Teitelman, A. M., Tennille, J., Bohinski, J., Jemmott, L. S., y Jemmott, J. B. (2013). Urban adolescent girls's perspectives on multiple partners in the context of the sexual double standard and intimate partner violence. Journal of the Association of Nurses in AIDS Care, 24, 308-321. https://dx.doi. org/10.1016/i.jana.2013.04.001

Turchik, J. A., y Gidycz, C. A. (2012). Prediction of sexual risk behaviors in college students using the theory of planned behavior: A prospective analysis. Journal of Social and Clinical Psychology, 31, 1-27. https://dx.doi. org/10.1521/iscp.2012.31.1.1

van Elm, E., Altman, D. G., Egger, M., Pocok, S. J., Gøtzschee, P. C., y Vandenbroucke, J. P. (2008). Declaración de la iniciativa STROBE (Strenghtening the Reporting of Observational studies in Epidemiology): Directrices para la comunicación de estudios observacionales [The Strengthening the Reporting of Observational Studies in Epidemiology [STROBE] statement: Guidelines for reporting observational studies]. Gaceta Sanitaria, 22, 144-150.

Yanez-Peñúñuri, L. Y., Martínez-Gómez, J. A., y Rey-Anacona, C. A. (2019). Therapeutic intervention for victims and perpetrators of dating violence: A systematic review. Revista Iberoamericana de Psicología y Salud, 10, 107-121. https://dx.doi.org/10.23923/i. rips.2019.02.029 